\title{
Dopamine Transporter Genotype and Methylphenidate Dose Response in Children with ADHD
}

\author{
Mark A Stein*,', Irwin D Waldman², Christopher S Sarampote ${ }^{3}$, Karen E Seymour ${ }^{3}$, Adelaide S Robb ${ }^{3}$, \\ Charles Conlon ${ }^{3}$, Soo-Jeong Kim' and Edwin H Cook Jr' \\ 'Department of Psychiatry, The University of Chicago, Chicago, IL, USA; '2Department of Psychology, Emory University, Atlanta, GA, USA; \\ ${ }^{3}$ Departments of Psychiatry and Pediatrics, Children's National Medical Center, Washington, DC, USA
}

\begin{abstract}
Stimulant medications, such as methylphenidate $(\mathrm{MPH})$, are the most commonly used, effective treatment for ADHD. MPH acts primarily by inhibiting the dopamine transporter (DAT), a protein responsible for the reuptake of dopamine from the synapse into presynaptic terminals. We sought to evaluate the relationship between DATI 3'-untranslated region (3'-UTR) variable number tandem repeats (VNTR) genotypes and dose response to $\mathrm{MPH}$. Children with $\mathrm{ADHD}(n=47)$, ages $5-16$ years (mean $=9.02$ years), underwent a 4-week, double-blinded, crossover trial with forced weekly dosage changes. Children were genotyped for the DATI VNTR and evaluated on placebo and three dosage levels of $O R O S^{\circledR}$ MPH. Parents and clinicians who were blind to genotype and medication status rated $A D H D$ symptoms, impairment, and stimulant side effects each week. Children who were homozygous for the less common, 9repeat DATI 3'-UTR genotype displayed a distinct dose-response curve from that of the other genotype groups, with an absence of typical linear improvement when the dose was increased from $18 \mathrm{mg}$ to 36 and $54 \mathrm{mg}$. Further research is needed to determine the mechanisms related to poor response in patients with the 9/9-repeat genotype, and to determine if this group responds differentially to alternative treatments.

Neuropsychopharmacology (2005) 30, I374-1382, advance online publication, I3 April 2005; doi: I0. I038/sj.npp. I 3007 I 8
\end{abstract}

Keywords: ADHD; dopamine transporter; pharmacogenetics; methylphenidate

\section{INTRODUCTION}

$\mathrm{ADHD}$ is one of the most common neuropsychiatric disorders of childhood and adolescence, and is associated with persistent impairments in academic and social adaptive functioning (Biederman et al, 1991; Stein et al, 1995; Zametkin, 1995; Barkley, 2002; Barkley et al, 2002). Current models of ADHD pathophysiology and stimulant response emphasize dysfunction in catecholamine systems (Castellanos and Tannock, 2002; Kirley et al, 2002). Methylphenidate (MPH) blocks the dopamine and norepinephrine transporters (Castellanos, 1997). Furthermore, neuroimaging studies have consistently reported abnormalities in nigrostriatal dopaminergic brain structures (Aylward et al, 1996; Castellanos et al, 1996) and function (Zametkin et al, 1993; Vaidya et al, 1998; Zametkin and Liotta, 1998) in children with ADHD. Consistent with these findings, neuroimaging studies in ADHD adults have shown

*Correspondence: Dr MA Stein, Department of Psychiatry, Child \& Adolescent Psychiatry, The University of Chicago, 584I S. Maryland Ave., Chicago, IL 60637, USA, Tel: + I 773834 9756, Fax: + I 847 242 065।, E-mail: mstein@yoda.bsd.uchicago.edu

Received 9 September 2004; revised 3 February 2005; accepted 7 February 2005

Online publication: I 5 February 2005 at http://www.acnp.org/citations/ NPP02 I 505040409/default.pdf alterations in presynaptic dopamine storage processes (Ernst et al, 1998) and in the density of striatal dopamine transporters (DATs) (Dougherty et al, 1999; Krause et al, $2000,2002)$, although not consistently.

Stimulant medications bind to the DAT, inhibiting reuptake and increasing synaptic dopamine (Volkow et al, 2002). The DAT gene (DAT1) was initially investigated as a primary candidate gene for ADHD susceptibility. Cook et al (1995) demonstrated an association between ADHD and the 10-repeat allele of a variable number tandem repeat (VNTR) in the $3^{\prime}$-untranslated region ( $3^{\prime}$-UTR) of the gene, a finding that has been replicated in many (Gill et al, 1997; Hawi et al, 2003; Waldman et al, 1998; Daly et al, 1999; Curran et al, 2001), but not in all samples (Palmer et al, 1999; Todd et al, 2001). To date, molecular genetic studies of ADHD have been limited by genetic and phenotypic heterogeneity (Todd, 2000). However, the association of the 10-repeat DAT1 allele with ADHD, coupled with the key role of the DAT in the mechanism of action of stimulants, suggests that DAT1 is a plausible candidate for predicting response to MPH (Masellis et al, 2002).

$\mathrm{MPH}$ - and amphetamine (AMP)-based stimulant medications are the most common medical treatments for ADHD (Elia et al, 1999; Conners, 2002), and at low to moderate dosage levels, there is generally an inverse linear doseresponse effect on ADHD symptoms (eg Douglas et al, 1986; 
Stein et al, 2003). In this paper, we report the results of a prospective, placebo-controlled study of DAT1 genotypes and dose-related $\mathrm{MPH}$ response. Outcome measures included both dimensional and categorical measures of ADHD symptoms, side effects, and impairment. Given that the majority of ADHD youth respond to stimulants and that the 10-repeat allele of DAT1 is the most common allele (Kang et al, 1999), we hypothesized that presence of the 10 -repeat allele would be associated with a positive response to $\mathrm{MPH}$.

\section{PATIENTS AND METHODS}

This study was reviewed and approved by the Institutional Review Boards of The University of Chicago, Children's National Medical Center, and the General Clinical Research Center Advisory Council. Participants were children, ages 5-16 years, who were referred to a suburban clinic specializing in $\mathrm{ADHD}$, which is affiliated with a large medical center. All participants completed a semistructured diagnostic interview conducted with the parents and child by a child and adolescent psychiatrist or psychologist (HALP diagnostic interview available from senior author), and met DSM-IV criteria for ADHD based upon the interview and a 'best estimate' diagnosis based upon a standardized 4-6h evaluation. Diagnostic procedures and subjects are described in more detail in a previous paper (Stein et al, 2003).

In all, 47 children were evaluated in a double-blind, placebo-controlled, crossover study with forced weekly titrations of three dose conditions $(18,36$, and $54 \mathrm{mg}$ ) of OROS $^{\circledR}$ MPH (Concerta). Children previously taking stimulant medications completed a 2 -week washout period prior to beginning the study. During the initial visit and at each weekly visit, children and their parents met with the clinical staff to discuss medication effects and to complete the ADHD Rating Scale-IV: Home Version (ADHD-RS) (DuPaul et al, 1998), and Stimulant Side Effect Scale (Barkley et al, 1990). In addition, Clinical Global Impression-Severity of Impairment (CGI-S) ratings were completed (Guy, 1976), which ranged from 1 ('no impairment, normal') to 7 ('maximal, profound impairment'). A positive response was characterized as resulting in a CGI-S score of $\leqslant 3$, indicating mild, slight, or no impairment.

\section{Genotyping}

DAT1 3'-UTR VNTR genotyping. DNA was extracted from whole blood with a PureGene kit from $10 \mathrm{ml}$ of whole blood. Genotyping was performed in the following manner. PCR was carried out in a $10 \mu \mathrm{l}$ volume containing $50 \mathrm{ng}$ of genomic DNA, $0.5 \mu \mathrm{M}$ of each primer, one of which was $5^{\prime}$ fluorescently labeled, $200 \mu \mathrm{M}$ of each dNTP (dATP, dCTP, dGTP, dTTP), $1 \times$ PCR buffer, $2 \mathrm{mM} \mathrm{MgCl}_{2}$, and $0.5 \mathrm{U} \mathrm{Taq}$ polymerase (Amplitaq Gold). Samples were amplified on a 9700 thermal cycler with an initial $12 \mathrm{~min}$ step to heat activate the enzyme, 40 cycles consisting of a denaturation step of $95^{\circ} \mathrm{C}$ for $30 \mathrm{~s}$, an annealing step of $68^{\circ} \mathrm{C}$ for $30 \mathrm{~s}$, and an extension step of $72^{\circ} \mathrm{C}$ for $30 \mathrm{~s}$. Products were injected on an ABI 3700 multicapillary array genetic analyzer with POP6 polymer. Alleles were called with GeneMapper software, blind to all phenotypic information.

\section{Statistical Plan}

The primary analyses of DAT1 genotype differences in dose-response effects were conducted using a growth-curve analytic approach as implemented via hierarchical linear modeling (HLM; Bryk and Raudednbush, 1992). In the HLM framework, the dose-response effects are handled in a firstlevel regression equation, in which an outcome variable is regressed on dosage, and the parameters of dose response (ie the linear and/or curvilinear effects of dosage on a particular outcome variable) are modeled as a function of explanatory variables in a second-level regression equation. In the present study, these explanatory variables included the child's DAT1 genotype, and the covariates sex, age, and ADHD diagnostic subtype. The first-level regression equation is shown below

$$
Y_{i j}=\beta_{0}+\beta_{1} X_{i 1}+\beta_{2} X_{i 2}^{2}+\cdots+\beta_{k} X_{i k}^{k}+\varepsilon_{i}
$$

where $Y_{i j}$ is the value of the outcome variable for the $i$ th individual at the $j$ th dosage level, $\beta_{0}$ is the regression constant, $X_{i j}$ is a particular dosage level for a particular individual, and $\beta_{k}$ is a regression coefficient representing a linear or curvilinear (eg quadratic, cubic) effect of dosage on the outcome variable. The correlates of these doseresponse parameters are modeled in second-level regression equations, an example of which is shown below

$$
\beta_{k}=\gamma_{0 j}+\gamma_{k j} C_{i k j}+v_{k}
$$

where $\beta_{k}$ represents a parameter of dose response (ie the linear and/or curvilinear slope of a particular outcome variable on dosage), $\gamma_{0 j}$ represents a regression constant, $C_{i k j}$ represents the value of a particular explanatory variable or covariate (eg DAT1 genotype, sex, age, and diagnostic subtype) for the $i$ th individual, and $\gamma_{k j}$ is a regression coefficient representing the effects of the explanatory variable or covariate, $C_{i k j}$, on the parameter of dose response, $\beta_{k}$. Note that one can use HLM to model multiple outcome variables simultaneously, or non-normally distributed outcome variables. This method results in greater precision in the estimation of both individual participants' dose-response curves, as well as the relation of dose response to explanatory variables.

This statistical approach has several advantages over more conventional methods. First, in contrast to repeated measures analysis of variance, HLM utilizes available data on all participants at all time points (ie dosage levels), such that individuals are included in the analysis even if they are missing data for one or more dosage levels. Second, in contrast to other methods that test for genotype differences in symptom and impairment levels between each pair of dosage levels in a piecemeal manner, HLM conducts a single analysis of genotype differences in dose response using all of the available data simultaneously. These features both increase statistical power due to the maximal inclusion of available data and minimize Type I error because far fewer statistical tests are conducted.

\section{RESULTS}

There was a strong male preponderance in this sample, as $70 \%$ (33) were male and 30\% (14) were female. Children ranged in age from 5 to 16 years with a mean age of 9.07 
years $(\mathrm{SD}=2.5)$. Additionally, $89 \%(42)$ of the subjects were Caucasian, 4\% (2) were African American, 2\% (1) were Hispanic, and 4\% (2) reported other ethnicities. With regard to $\mathrm{ADHD}$ subtype, $68 \%$ (32) met criteria for $\mathrm{ADHD}$ Combined Type (ADHD-CT), while $32 \%$ (15) were diagnosed with ADHD-Predominately Inattentive Type (ADHD-PI). In addition, $17 \%$ met criteria for Oppositional Defiant Disorder, $11 \%$ displayed encopresis/enuresis, and $2 \%$ displayed a tic disorder. In all, $70 \%$ of participants were stimulant naive (33), whereas $30 \%$ (14) had previously been treated with a stimulant according to parent report.

Genotype frequencies for the 47 children were as follows: six $(13 \%)$ were homozygous for the DAT1 9-repeat allele, $22(46 \%)$ had one copy of the 9-repeat and one copy of 10 -repeat allele, and $19(42 \%)$ were homozygous for the 10 -repeat allele. The DAT1 genotype distribution of the total sample was in Hardy-Weinberg equilibrium $\left(\chi^{2}=0.009\right.$, NS).

Demographic and descriptive characteristics of the sample are reported in Table 1 . There were no significant DAT1 genotype group differences in demographic characteristics such as age, gender, or ethnicity, or in previous stimulant history. Additionally, there were no significant DAT1 genotype-group differences in ADHD symptoms as measured by the ADHD RS, diagnostic subtype, stimulant medication history, WISC-III IQ, WIAT achievement, CBCL internalizing and externalizing psychopathology, or CGI-S scores.

We examined whether changes in ADHD symptoms, as a function of dosage level, varied by DAT1 genotype, and then re-examined this using ADHD diagnostic subtype, sex, and age as covariates. ADHD symptom levels as a function of OROS MPH dosage varied significantly by genotype in the HLM growth-curve analyses $(p=0.030)$. Differences in parent ratings of ADHD symptoms as a function of OROS $\mathrm{MPH}$ dosage also varied by $\mathrm{ADHD}$ diagnostic subtype (0.018), and the DAT1 genotype differences in dose response were stronger when diagnostic subtype was used as a covariate $(p=0.013)$. Sex and age were not related to dose-response effects on ADHD symptoms, or on most of the other outcome measures, and thus were not used as covariates in the analysis of DAT1 genotype differences in dose response for most of the dependent variables (with the exception of Total Stimulant Side Effects, to be discussed below). Total ADHD RS score differences in dose response as a function of DAT1 genotype are shown in Figure 1 below.

Differences in dose response by DAT1 genotype were significant for Inattentive symptoms $(p=0.050)$ and represented a statistical trend for Hyperactive-Impulsive symptoms $(p=0.088)$. As portrayed in Figures 2 and 3 below, when ADHD diagnostic subtype was included as a covariate, dose response for both dimensions of $\mathrm{ADHD}$ symptoms differed significantly by DAT1 genotype (both $p$ 's $=0.037$ ).

Dose-response differences in CGI impairment ratings also varied significantly and substantially by DAT1 genotype ( $p=0.006$, see Figure 4 below), and became stronger when ADHD diagnostic subtype was used as a covariate $(p=0.002)$.

As a categorical measure of positive response, we used a cutoff score of $\leqslant 3$ (ie 'minimal, mild, or no impairment') on the CGI-S and contrasted children with no copies of the
DAT1 10-repeat allele (ie homozygous for 9-repeat allele) to children with one or two copies of the 10-repeat allele on this index at placebo and at each dosage level (see Figure 5 below). While the two DAT1 genotype groups did not differ in CGI-S ratings during placebo or at the low-dose condition $(p=0.174$ and 0.381 , respectively), the two groups differed marginally or significantly at the 36 and $54 \mathrm{mg}$ doses ( $p=0.063$ and 0.004 , respectively). Odds ratios contrasting the proportion of children who experienced reduction of impairment with one or two copies vs no copies of the DAT1 10-repeat allele increased from low to medium to high dosage levels (ORs $=1.30,1.72$, and 2.64, respectively). These results were nearly identical controlling for the covariates sex, age, and ADHD diagnostic subtype, and the odds ratio for the high-dose level increased somewhat with the inclusion of these covariates $(\mathrm{OR}=3.60$ vs 2.64). At the highest dose condition $(54 \mathrm{mg})$, $75 \%$ of those with one copy of the 10 -repeat and $87 \%$ of those with two copies of the 10-repeat allele displayed minimal or no impairment as compared to only $20 \%$ of those homozygous for the 9 -repeat allele $\left(\chi^{2}=6.92, \mathrm{df}=1\right.$, $p<0.01)$.

We also examined whether a different, more stringent cutoff score for treatment success would affect the findings, and thus analyzed the relationship between $D A T 1$ genotypes and response as defined by a CGI-S score of $\leqslant 2$, which is analogous to a remission standard. At the $36 \mathrm{mg}$ dose condition, $48 \%$ of those with one or two copies of the 10 repeat displayed minimal or no impairment as compared to only $16 \%$ of those homozygous for the 9-repeat allele. At the $54 \mathrm{mg}$ dose condition, $57 \%$ of those with one or two copies of the 10-repeat allele met the remission standard as compared to none of those with the 9/9 genotype. These results suggest increasing dose-related reduction in impairment for children with one or two copies of the DAT1 10-repeat allele, while those homozygous for the 9-repeat were much less likely to display dose-related improvement or remission.

Finally, we examined dose-response effects on parent ratings of stimulant side effects. The nature of these sex and age differences was that total side effects were higher for boys than girls, and for younger than older children, at all dosage levels. DAT1 genotype differences in dose-response effects on total side effects emerged in the HLM analyses when sex and age were entered as covariates $(p=0.034$, see Figure 6 below).

\section{DISCUSSION}

Utilizing a prospective design with dimensional and categorical measures of ADHD symptoms, impairment, and side effects, DAT1 genotype differences in dose response were such that levels of ADHD symptoms and impairment decreased in a linear manner, and total side effects increased, as a function of increasing OROS MPH dosage levels for children with one or two copies of the 10-repeat allele. In contrast, youth homozygous for the less common, 9-repeat allele displayed a markedly poor response to $\mathrm{MPH}$ at doses typically associated with response to MPH. This suggests a recessive effect of the 9-repeat allele on the phenotype of stimulant nonresponse. 
Table I Demographic and Descriptive Characteristics by DATI Genotype

Number of DATI 10-repeat alleles

\begin{tabular}{cccc}
\hline $\mathbf{0}(\boldsymbol{n}=\mathbf{6})$ & $\mathbf{I}(\boldsymbol{n}=\mathbf{2 2})$ & $\mathbf{2}(\boldsymbol{n}=\mathbf{1 9 )}$ & Total $(\boldsymbol{n}=\mathbf{4 7})$ \\
$9.17(3.06)$ & $9.09(2.37)$ & $8.89(2.54)$ & $9.02(2.47)$ \\
$6-14$ & $6-16$ & $5-15$ & $5-16$
\end{tabular}

Gender

Male (\%)

Female (\%)

Ethnicity

Caucasian (\%)

African-American (\%)

Hispanic (\%)

Other (\%)

\section{ADHD diagnosis}

Combined Type (\%)

Inattentive Type (\%)

5 (83.3)

। (16.7)

$4(66.7)$

$2(33.3)$

No previous treatment
Previous treatment (\%)

ADHD-RS

Mean (SD)

Range

CBCL internalizing $T$-score

Mean (SD)

$62.17(11.21)$

$54.19(13.98)$

$52.78(\mid 1.41)$

CBCL externalizing T-score

Mean (SD)

$57.33(11.13)$

57.1 (9.09)

$56.06(10.43)$

\section{CGI-S score}

Mean (SD)

WISC-III FSIQ

Mean (SD)

Range

WIAT scale scores

Word reading

$$
\text { Mean (SD) }
$$

Range

Mathematics reasoning

$$
\begin{aligned}
& \text { Mean (SD) } \\
& \text { Range } \\
& \text { Spelling } \\
& \text { Mean (SD) } \\
& \text { Range }
\end{aligned}
$$$$
\text { Spelling }
$$

4.I (0.83)

$$
109.9(17.6)
$$$$
74-148
$$

$93-131$

$101.8(10.62)$

$88-112$

$102.2(13.16)$

$82-114$

$95.2(7.95)$

89-109
107.33 (15.95)

74-140

108.24 ( | I.92)

$84-128$

102.8| (1 1.73)

$78-130$
103.86 (13.95)

$72-126$

$103.29(9.76)$

$89-128$

$104.29(12.93)$

$88-135$
$54.69(12.75)$

$56.71(9.69)$

$33(70.2)$

14 (29.8)

42 (89.4)

2 (4.3)

I (2.1)

$2(4.3)$

32 (68. I)

15 (31.9)

$33(70.2)$

14 (29.8)

$32.7(9.61)$

9-49

$4.37(0.93)$

106.75 (16.54)
68-148
68-130

105.43 (14.54)

72-140

105.75 ( 11.38$)$

82-128

102.38 ( 11.86$)$

78-138 
Number of DATI 10-repeat alleles

\begin{tabular}{|c|c|c|c|c|}
\hline & & & & \multirow[b]{2}{*}{ Total $(n=47)$} \\
\hline & $0(n=6)$ & I $(n=22)$ & $2(n=19)$ & \\
\hline \multicolumn{5}{|c|}{ Reading comprehension } \\
\hline Mean (SD) & $103(15.28)$ & $109.25(15.42)$ & $102.5(25.42)$ & $106.43(18.5)$ \\
\hline \multicolumn{5}{|c|}{ Numerical operations } \\
\hline Mean (SD) & $98.2(10.33)$ & $103(12.67)$ & $99(13.09)$ & $101.05(12.43)$ \\
\hline Mean (SD) & $99(17.38)$ & $105.15(11.55)$ & $102.5(\mid 1.57)$ & $103.58(12.03)$ \\
\hline Range & $76-117$ & $85-124$ & $82-116$ & $76-124$ \\
\hline \multicolumn{5}{|l|}{ Oral expression } \\
\hline Mean (SD) & $112(7.53)$ & I09.37 (I 15.82) & I09.08 (| 4.06$)$ & I09.57(14.22) \\
\hline Range & $106-123$ & $76-146$ & $80-127$ & $76-146$ \\
\hline
\end{tabular}

$\mathrm{CBCL}=$ Child Behavior Checklist; CGI = Clinical Global Impressions Scale; WISC-III = Wechsler Intelligence Scale for Children-Third Edition; WIAT = Wechsler Individual Achievement Test.

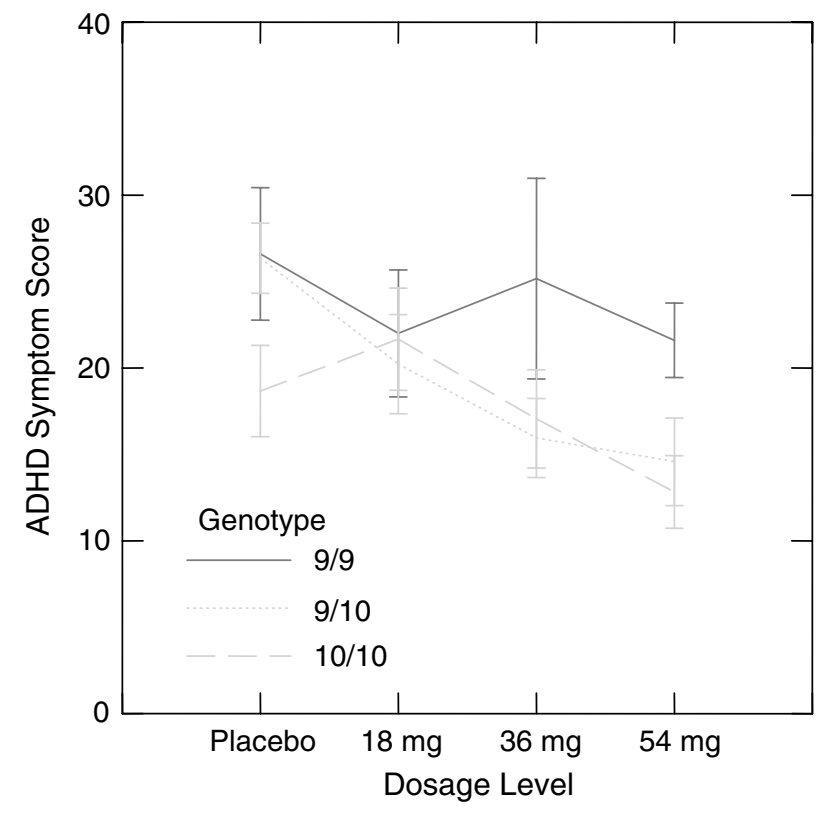

Figure I ADHD symptom levels (with SE) by dosage level by genotype.

The association of genotypes containing the 10-repeat allele with a positive response to MPH is in agreement with the findings of Kirley et al (2003), but differs from the Winsberg and Cummings and Roman et al studies (Winsberg and Cummings, 1999; Roman et al, 2001). Kirley et al (2003) reported that transmission of the 10-repeat allele was significantly greater in children who displayed a 'very good' retrospectively rated response to MPH as compared to those with 'mediocre' or 'no response' in a sample of 117 Irish school children. In contrast, Winsberg

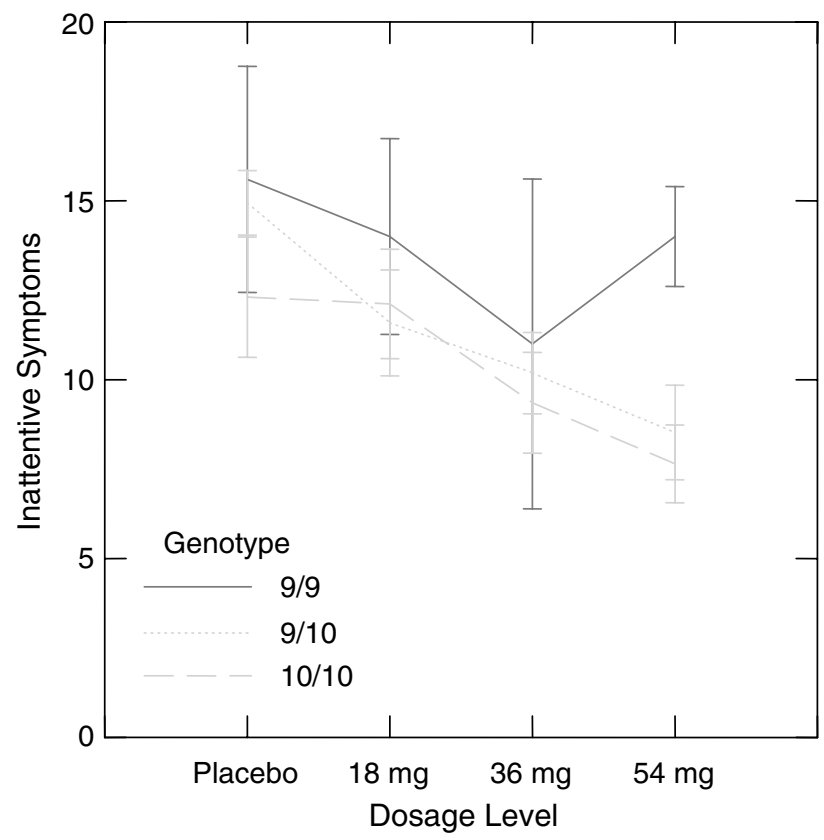

Figure 2 Inattentive symptom levels (with SE) by dosage level by genotype.

and Cummings (1999) reported that $86 \%$ of 14 'poor' responders (defined as less than a 50\% reduction in ADHD symptoms on parent ratings) were homozygous for the 10repeat allele, as compared to $31 \%$ of 16 'positive' responders in a sample of 30 stimulant naive African-American children with ADHD. Using a similar methodology but with a Brazilian sample, Roman et al (2001) reported that $47 \%$ of those with the $10 / 10$ genotype demonstrated a $50 \%$ 


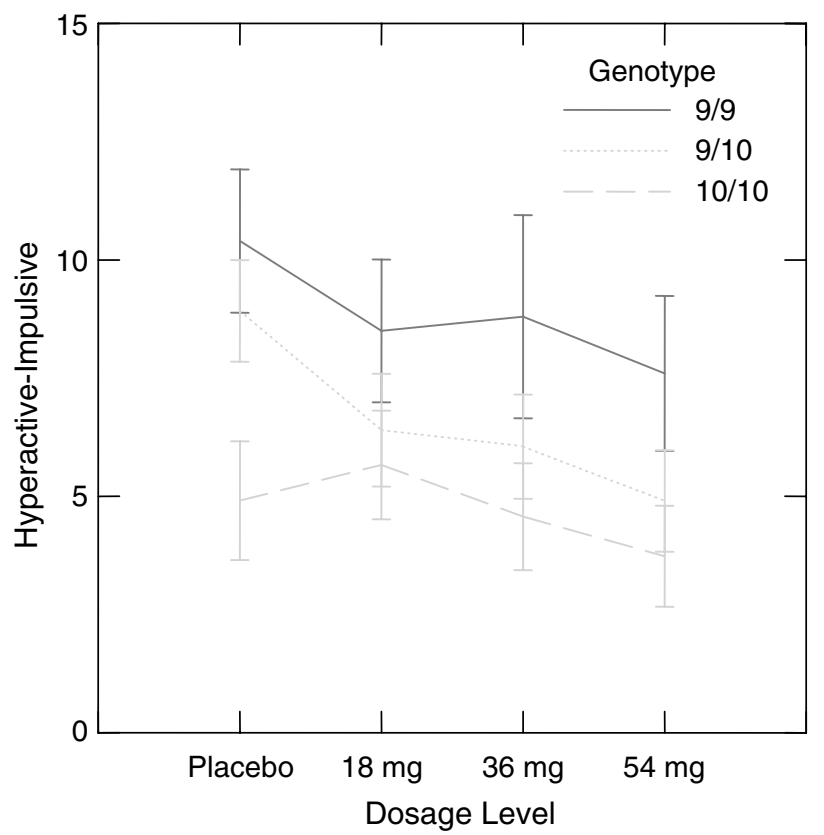

Figure 3 Hyperactive-Impulsive symptom levels (with SE) by dosage level by genotype.

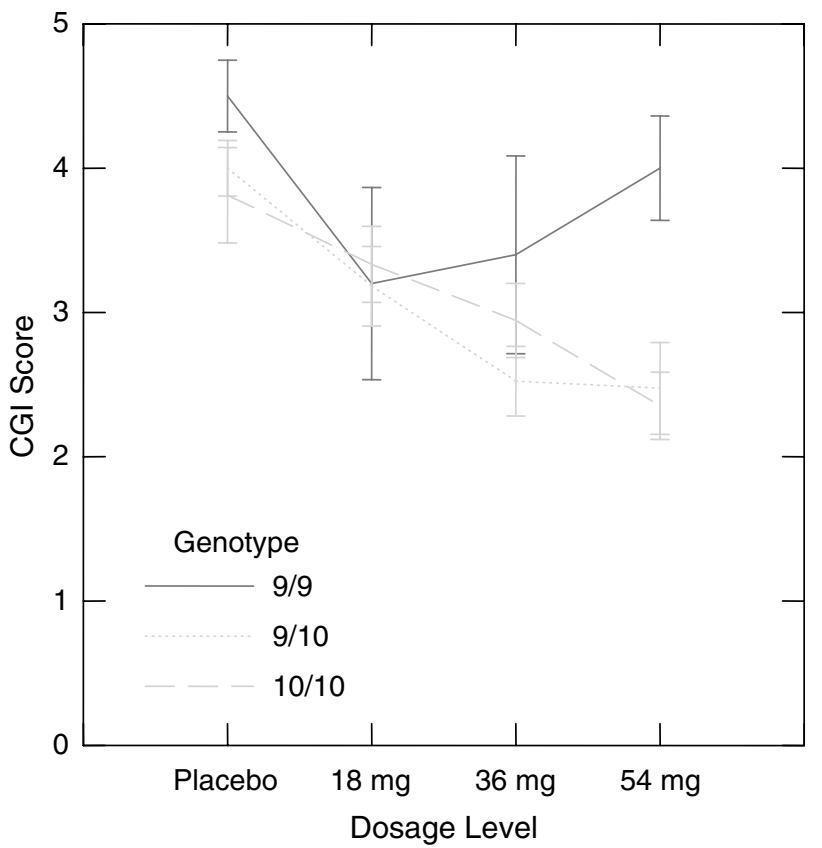

Figure $4 \mathrm{CGI}-\mathrm{S}$ Levels (with SE) by dosage level by genotype.

reduction in ADHD ratings, as compared to $75 \%$ of $\mathrm{ADHD}$ youth with the $9 / 10$ and $9 / 9$ genotypes included in the same group (Roman et al, 2001).

Indeed, there are numerous methodological differences between these early pharmacogenetic studies and the current study, which could account for different findings, including study design factors (prospective $v s$ retrospective or naturalistic), duration of trial, treatment regimen and dose, and differences in sample characteristics (eg ethnicity,

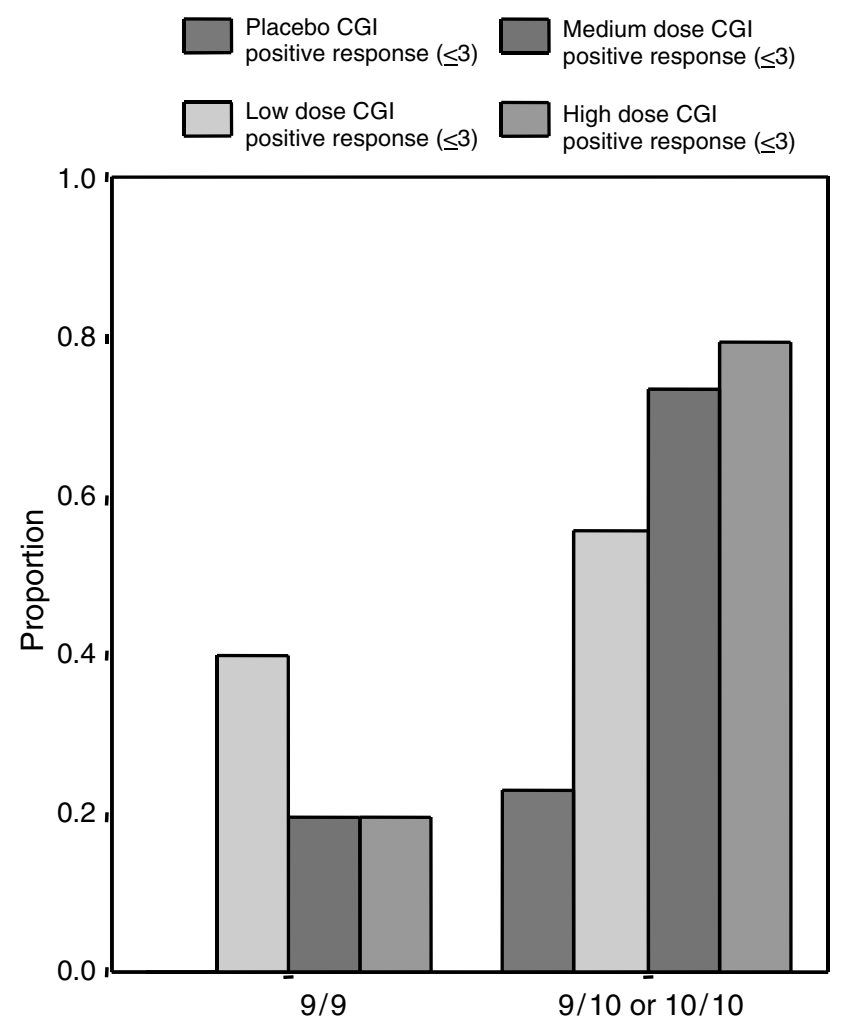

Figure 5 Proportion attaining $\mathrm{CGl} \leqslant 3$ in those with and without 10-repeat allele.

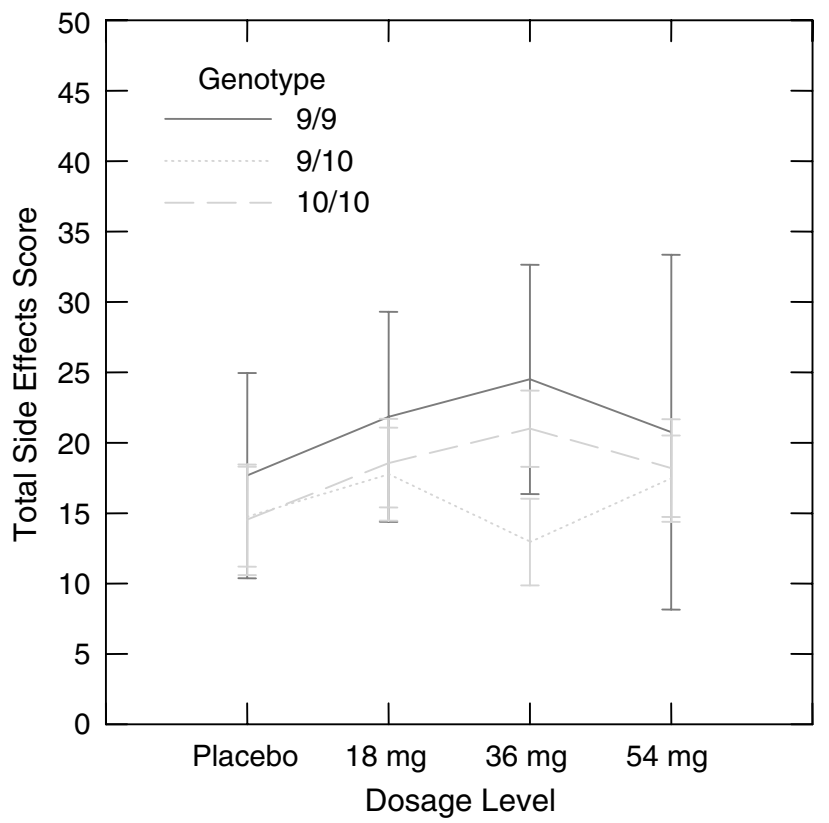

Figure 6 Total side effects levels (with SE) by dosage level by genotype.

previous medication history, ADHD subtype). Most importantly, the previously published pharmacogenetic studies of DAT1 assumed a dominant effect of the 9-repeat allele. The present study is the first pharmacogenetic study of ADHD, 
which analyzed the less common 9/9-repeat genotype group separately, without combining it with the more prevalent 9/ 10-repeat genotype group.

Another difference between ADHD pharmacogenetic studies is how the phenotype of stimulant response $v s$ nonresponse was defined. Rather than relying on a single measure or arbitrary cutoff score, we examined dose-response curves for measures of ADHD symptoms, impairment, and stimulant side effects. (We also reported MPH response based upon CGI-S impairment ratings using two different cutoff scores for heuristic purposes.) An additional benefit of the dose-response paradigm is that response to multiple dosages are examined, providing several opportunities to respond rather than relying on a single dose or a clinically determined dose, which may vary significantly due to individual clinician dosing strategies.

The 9-repeat is the second most common allele after the 10-repeat, occurring in approximately $20-30 \%$ of US and European populations (Kang et al, 1999). Additional support for a recessive effect of the 9-repeat allele on stimulant response is provided by the recent findings of Lott et al (2003) and Kirley (2004). Lott et al (2003) reported that healthy college students homozygous for the 9-repeat allele differed from students with the other DAT1 genotypes containing the 10-repeat allele in their perception of AMP effects. Specifically, individuals with the 9/9 genotype showed significantly less endorsement of 'feels drug' in response to AMP. Kirley (2004) recently reported additional analysis of their study sample (Kirley et al, 2003). Consistent with the present study, a $25 \%$ response rate to $\mathrm{MPH}$ was reported in individuals with no copies of the 10-repeat allele as compared to $65 \%$ children with one or two copies of the 10-repeat allele. Thus, when the $9 / 9$ is not combined with the $9 / 10$ genotype, there appears to be a clear difference in stimulant response. In addition, there are also studies reporting atypical stimulant response in individuals with the 9-repeat allele in other patient groups (see Gelernter et al, 1994; Ujike et al, 2003).

The biological mechanism by which the 9/9 genotype might lead to an atypical response to CNS stimulants is presently unknown. The DAT1 $3^{\prime}$-UTR VNTR may be functional, or may be in high linkage disequilibrium with a functional variant, and consequently predict stimulant response. Heinz et al (2000) reported that individuals with the 9-/10-repeat genotype had a $22 \%$ reduction of DAT protein availability in putamen compared to $10 / 10$ homozygous individuals, and speculated that stimulant effects would be most pronounced in the $10 / 10$ homozygous individuals for whom DAT protein appears to be more abundant. These findings were not replicated in two other studies (Jacobsen et al, 2000; Martinez et al, 2001). Indeed, the contrast of $9 / 10$ with $10 /$ 10 genotypes is not where our data suggest the most interesting question is, but rather between $9 / 9$ vs $9 / 10$ and $10 / 10$ genotypes. Of most relevance, Mill et al (2002) demonstrated decreased DAT1 expression associated with the 9/9 genotype relative to genotypes containing the 10 repeat allele in human brain tissue and lymphocytes. As suggested by Kirley et al (2003), these studies provide intriguing clues regarding the relationship between varia- bility in the length or sequence of the $3^{\prime}$-UTR of the DAT1 gene and levels of DAT1 in the brain, which will hopefully lead to more basic studies than can attempt to identify specific mechanisms.

The statistical significance of the effect of the $9 / 9$ genotype on $\mathrm{MPH}$ response is strong given the relatively modest sample size. However, replication attempts in larger samples are necessary for the results to be more conclusive, due to the relative scarcity of children with the $9 / 9$ genotype. If replicated, further studies of individuals homozygous 9-repeat allele are indicated, with the goal of determining clinical predictors of nonresponse, specific adverse events, or perhaps neuropsychological or neurophysiologic endophenotypes (eg Loo et al, 2003). An additional question is whether those with the 9/9 genotype respond to either an alternative stimulant (eg AMP or mixed AMP salts) or nonstimulant treatment (eg atomoxetine), which appears to work through a different mechanism than do stimulants. Determination of positive predictive value and negative predictive value will be important in determining the clinical utility of pretreatment genotyping in ADHD.

Pharmacogenetic study of a locus with replicated (but not consistent and fully confirmed) evidence for family-based association with the same disorder raises interesting issues. For example, if the $9 / 9$ genotype is associated with nonresponse, tertiary clinic-based samples selected for family-based association of DAT1 with ADHD may lead to study of nonrepresentative samples and lead to bias of transmission against the 10-repeat allele. Conversely, if the $10 / 10$ genotype is actually more common in ADHD, there may be fewer $9 / 9$ genotypes present in representative samples of ADHD. Ascertainment is critical in all studies, and it is important to note that the current sample was not selected on the basis of genotype or past history of stimulant responsiveness.

Pharmacogenetic studies of ADHD are in a relatively early stage, but hold considerable promise for this disorder (Rohde et al, 2003). Although it is true that the majority of individuals with ADHD initially respond positively to stimulants, long-term effectiveness is much more modest (Charach et al, 2004). Being able to predict which individuals are likely to respond positively or which individuals do not tolerate stimulants would alter the trial and error approach to stimulant treatment of ADHD. Ultimately, it is hoped that further understanding of the genetic variability in ADHD treatment response may shed light on both genetic and nongenetic factors, which contribute to outcome. This may ultimately guide and improve future treatment decisions and assist in targeted development of alternative pharmacological strategies for nonresponders.

\section{ACKNOWLEDGEMENTS}

This study was supported by National Institute of Mental Health Grant K24MHO1823 and by MO1-RR13297 from the General Clinical Research Center Program of the National Center for Research Resources. We thank the patients and their families who participated in the study and their referring physicians. 


\section{REFERENCES}

Aylward EH, Reiss AL, Reader MJ, Singer HS, Brown JE, Denckla MB (1996). Basal ganglia volumes in children with attentiondeficit hyperactivity disorder. J Child Neurol 11: 112-115.

Barkley R, McMurray M, Edelbrock C, Myers K (1990). Side effects of $\mathrm{MPH}$ in children with attention deficit hyperactivity disorder: a systematic placebo controlled evaluation. Pediatrics 86: 184-192.

Barkley RA (2002). International Consensus Statement on ADHD. J Abnorm Child Psychol 41: 1389.

Barkley RA, Fischer M, Smallish L, Fletcher K (2002). The persistence of attention-deficit/hyperactivity disorder into young adulthood as a function of reporting source and definition of disorder. J Abnorm Psychol 111: 279-289.

Biederman JJ, Newcorn J, Sprich S (1991). Comorbidity of attention deficit hyperactivity disorder with conduct, depressive, anxiety, and other disorders. Am J Psychiatry 148: 564-577.

Bryk AT, Raudednbush S (1992). Hierarchical Linear Models: Applications and Data Analysis Methods. Sage: Newbury Park, CA.

Castellanos FX (1997). Toward a pathophysiology of attentiondeficit/hyperactivity disorder. Clin Pediatr 36: 381-393.

Castellanos FX, Giedd JN, Marsh WL, Hamburger SD, Vaituzis AC, Dickstein DP et al (1996). Quantitative brain magnetic resonance imaging in attention-deficit hyperactivity disorder. Arch Gen Psychiatry 53: 607-616.

Castellanos XF, Tannock R (2002). Neuroscience of attentiondeficit/hyperactivity disorder: the search for endophenotypes. Nat Rev Neurosci 8: 617-628.

Charach AA, Ickowicz A, Schachar R (2004). Stimulant treatment over five years, adherence, effectiveness, and adverse events. J Am Acad Child Adolesc Psychiatry 43: 559-567.

Cook EH, Stein MA, Krasowski MD, Cox NJ, Olkon DM, Kieffer JE et al (1995). Association of attention deficit disorder and the dopamine transporter gene. Am J Human Genet 56: 993-998.

Conners CK (2002). Forty years of methylphenidate treatment in attention-deficit/hyperactivity disorder. J Atten Disord 6: S17-S30.

Curran S, Mill J, Tahir E, Kent L, Richards S, Gould A et al (2001). Association study of a dopamine transporter polymorphism and attention deficit hyperactivity disorder in UK and Turkish samples. Mol Psychiatry 6: 425-428.

Daly G, Hawi Z, Fitzgerald M, Gill M (1999). Mapping susceptibility loci in attention deficit hyperactivity disorder: preferential transmission of parental alleles at DAT1, DBH and DRD5 to affected children. Mol Psychiatry 4: 192-196.

Dougherty DD, Bonab AA, Spencer TJ, Rauch SL, Madras BK, Fischman AJ (1999). Dopamine transporter density in patients with attention deficit hyperactivity disorder. Lancet 354: 2132-2133.

Douglas V, Barr R, Oneill ME, Britton BG (1986). Short term effects of methylphenidate on the cognitive, learning, and academic performance of children with attention deficit disorder in the laboratory and the classroom. J Child Psychol Psychiatry 27: 191-211.

DuPaul GJ, Power TJ, Anastopoulos AD (1998). ADHD Rating Scale-IV: Checklists, Norms, and Clinical Interpretations. Guilford Press: New York.

Elia J, Ambrosini PJ, Rapoport JL (1999). Treatment of attention deficit hyperactivity disorder. $N$ Engl J Med 349: $780-788$

Ernst M, Zametkin AJ, Mtochik JA, Jons PH, Cohen RM (1998). DOPA decarboxylase activity in attention deficit hyperactivity disorder adults. A [fluorine-18]fluorodopa positron emission tomographic study. J Neurosci 18: 5901-5907.

Gelernter J, Kranzler HR, Satel SL, Rao PA (1994). Genetic association between dopamine transporter protein alleles and cocaine-induced paranoia. Neuropsychopharmacology 11: 195-200.

Gill M, Daly G, Heron S, Haw Z, Fitzgerald M (1997). Confirmation of association between attention deficit hyperactivity disorder and a dopamine transporter polymorphism. Mol Psychiatry 2: 311-313.

Guy W (1976). ECDU Assessment Manual for Psychopharmacology, Revised. US Department of Health, Education, and Welfare: Bethesda, MD.

Hawi ZN, Lowe N, Kirley A, Gruenhage F, Nothen M, Greenwood T et al (2003). Linkage disequilibrium mapping at DAT1, DRD5 and $\mathrm{DBH}$ narrows the search for ADHD susceptibility alleles at these loci. Mol Psychiatry 8: 299-308.

Heinz A, Goldman D, Jones DW, Palmour R, Hommer D, Gorey JG et al (2000). Genotype influences in vivo dopamine transporter availability in human striatum. Neuropsychopharmacology 22: 133-139.

Jacobsen LK, Staley JK, Zoghibi SS, Seibyl JP, Kosten TR, Innis RB et al (2000). Prediction of dopamine transporter binding availability by genotype: a preliminary report. Am J Psychiatry 157: $1700-1703$.

Kang AM, Palmatier MA, Kidd KK (1999). Global variation of a 40-bp VNTR in the $3^{\prime}$-untranslated region of the dopamine transporter gene (SLC6A3). Biol Psychiatry 36: 150-160.

Kirley A (2004). The dopamine transporter gene and methylphenidate response in ADHD. 16th World Congress of the International Association for Child and Adolescent Psychiatry and Allied Professions (IACAPAP), August, Berlin.

Kirley A, Hawi Z, Daly G, McCarron M, Mullins C, Millar N et al (2002). Dopaminergic system genes in ADHD: toward a biological hypothesis. Neuropsychopharmacology 27: 607-619.

Kirley A, Lowe N, Hawi Z, Mullins C, Daly G, Waldman I et al (2003). Association of the $480 \mathrm{bp}$ DAT1 allele with methylphenidate response in a sample of Irish children with ADHD. Am J Med Genet 121: 50-54.

Krause KH, Dresel S, Krause J, Kung HF, Tatsch K, Lochmuller H (2002). Elevated striatal dopamine transporter in a drug naive patient with Tourette syndrome and attention deficit/hyperactivity disorder: positive effect of methylphenidate. J Neurol 249: 1116-1118.

Krause KH, Dresel SH, Krause J, Kung HF, Tatsch K (2000). Increased striatal dopamine transporter in adult patients with attention deficit hyperactivity disorder: effects of methylphenidate as measured by single photon emission computed tomography. Neurosci Lett 285: 107-110.

Loo SK, Specter E, Smolen A, Hopfer C, Teale PD, Reite ML (2003). Functional effects of the DAT1 polymorphism on EEG measures in ADHD. J Am Acad Child Adolesc Psychiatry 42: 986-993.

Lott D, Kim S, Cook EH, deWitt H (2003). Dopamine transporter genotype and amphetamine response. Neuropsychopharmacology 30: 602-609.

Martinez D, Gelernter J, Abi-Dargham A, van Dyck CH, Kegeles L, Innis RB et al (2001). The variable number of tandem repeats polymorphisms of the dopamine transporter gene is not associated with significant change in dopamine transporter phenotype in humans. Neuropsychopharmacology 24: 553-560.

Masellis M, Basile VS, Muglia P, Ozdemir V, Macciardi FM, Kennedy JL (2002). Psychiatric pharmacogenetics: personalizing psychostimulant therapy in attention-deficit/hyperactivity disorder. Behav Brain Res 130: 85-90.

Mill JP, Asherson P, Browes C, D’Souza U, Craig I (2002). Expression of the dopamine transporter gene is regulated by the 3 ' UTR VNTR: evidence from brain and lymphocytes using quantitative RT-PCR. Am J Med Genet 114: 975-979.

Palmer CG, Bailey JN, Ramsey C, Cantwell D, Sinsheimer JS, Del'Homme M et al (1999). No evidence of linkage or linkage disequilibrium between DAT1 and attention deficit hyperactivity disorder in a large sample. Psychiatr Genet 9: 157-160. 
Rohde LA, Roman T, Hutz MH (2003). Attention-deficit hyperactivity disorder: current aspects on pharmacogenetics. Pharamacogenom J 1: 1-3.

Roman T, Schmitz M, Polanczyk G, Eizirik M, Rohde LA, Hutz MH (2001). Attention-deficit hyperactivity disorder: a study of association with both the dopamine transporter gene and the dopamine D4 receptor gene. Am J Med Genet 105: 471-478.

Stein MA, Sarampote C, Waldman I, Robb AS, Conlon C, Pearl PL et al (2003). A dose-response study of OROS methylphenidate in children with attention-deficit/hyperactivity disorder. Pediatrics 112: e404-e413.

Stein MA, Szumowski E, Blondis TA, Roizen N (1995). Adaptive skills dysfunction in ADD and ADHD children. J Child Psychol Psychiatry 36: 663-670.

Todd RD (2000). Genetics of attention deficit hyperactivity disorder: are we ready for molecular genetic studies? Am J Med Genet 96: 241-243.

Todd RD, Jong YJ, Lobos EA, Reich W, Heath AC, Neuman RJ (2001). No association of the dopamine transporter gene $3^{\prime}$ VNTR polymorphism with ADHD subtypes in a population sample of twins. Am J Med Genet 105: 745-748.

Ujike $\mathrm{H}$, Harano M, Inada T, Yamada M, Komiyama T, Sekine Y et al (2003). Nine or fewer repeat alleles in VNTR polymorphism of the dopamine transporter gene is a strong risk factor for prolonged methamphetamine psychoses. Pharamacogen $\mathrm{J}$ 3: 242-247.

Vaidya CJ, Austin G, Kirkorian G, Ridelhuber HW, Desmond JE, Glover GH et al (1998). Selective effects of methylphenidate in attention deficit hyperactivity disorder: a functional magnetic resonance study. Proc Natl Acad Sci USA 95: 14494-14499.

Volkow ND, Wang G, Fowler JS, Logan J, Franchesi D, Maynard L et al (2002). Mechanism of action of methylphenidate: insights from PET imaging studies. J Atten Disord 6(Suppl 1): S31-S43.

Waldman ID, Rowe DC, Abramowitz A, Kozel ST, Mohr JH, Sherman SL et al (1998). Association and linkage of the dopamine transporter gene and attention-deficit hyperactivity disorder in children. Am J Hum Genet 63: 1767-1776.

Winsberg BG, Cummings DE (1999). Association of the dopamine transporter gene (DAT1) with poor methylphenidate response. J Am Acad Child Adolesc Psychiatry 38: 1474-1477.

Zametkin AJ (1995). Attention-deficit disorder. Born to be hyperactive? JAMA 273: 1871-1874.

Zametkin AJ, Liebenauer LL, Fitzgerald GA, King AC, Minkunas DV, Herscovitch P et al (1993). Brain metabolism in teenagers with attention-deficit hyperactivity disorder. Arch Gen Psychiatry 50: 333-340.

Zametkin AJ, Liotta W (1998). The neurobiology of attentiondeficit/hyperactivity disorder. J Clin Psychiatry 59(Suppl 7): 17-23. 